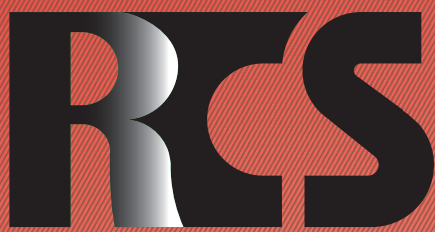

Depósito legal ppi $201502 Z U 4662$

Esta publicación científica en formato digital es continuidad de la revista impresa Depósito Legal: pp $197402 Z$ Z789

- ISSN: 1315-9518 • ISSN-E: 2477-9431

Revista de Ciencias Sociales

Universidad del Zulia. Revista de la Facultad de Ciencias Económicas y Sociales Vol. XXVII,

No. 4,2021

Esta publicación científica en formato digital es continuidad de la revista impresa Depósito Legal: pp $197402 Z 1789$ ISSN: $1315-9518$ 


\title{
Habilidades digitales e interés por estudiar en la modalidad E-Learning en estudiantes de Bachillerato*
}

\author{
Ramírez García, Adán Guillermo** \\ Rodríguez Sauceda, Elvia Nereyda ${ }^{* * *}$ \\ Pirela Hernández, Ana Arelis**** \\ Castillo Escalante, Irma Cecilia ${ }^{* * * * *}$
}

\begin{abstract}
Resumen
La educación es más competitiva cada día y en auge hacia la educación virtual en estos tiempos de pandemia, lo que ha obligado a estar a la vanguardia en todos los procesos de innovación educativa. El objetivo del presente estudio fue evaluar las habilidades digitales a partir del conocimiento autopercibido y el interés por estudiar en la modalidad e-learning de los estudiantes del Centro de Bachillerato Tecnológico Agropecuario 197, México. Se realizó una investigación descriptiva cuantitativa, la muestra se compuso de 175 individuos, el cuestionario fue una adaptación de la Matriz de Habilidades Digitales propuesta por la Universidad Nacional Autónoma de México, con 54 reactivos y se aplicó a través de la plataforma Aplicaciones de Google Formularios. Entre los resultados encontrados destaca que, al autoevaluarse sobre sus habilidades digitales, los estudiantes se perciben con un nivel de aceptable a muy bueno, sin embargo, muestran poco interés por los estudios en modalidad e-learning debido a la deficiente conectividad, baja calidad de la señal de internet, las metodologías y medios de enseñanza, así como las malas experiencias previas. Se concluye que, este tipo de estudios permite vislumbrar estrategias de alfabetización tecnológica que faciliten a los estudiantes avanzar hacia la sociedad del conocimiento.
\end{abstract}

Palabras clave: Competencias digitales; autopercepción; evaluación; habilidades digitales, TIC.

* Agradecimiento por la realización de este estudio a la Universidad Autónoma Chapingo, Subdirección de Planes y Programas de Estudio, Centro de Educación Continua, Dirección General Académica, así como a Patronato Universitario.

** Doctor en Geografía. Docente en la Universidad Autónoma Chapingo, México. E-mail: gramirezg@ taurus.chapingo.mx (iD ORCID: http://orcid.org/0000-0002-1711-5942

*** Doctora en Desarrollo Sustentable de Los Recursos Naturales. Docente en la Universidad Autónoma Indígena de México, México. E-mail: elviaro@uaim.edu.mx iD ORCID: https://orcid.org/0000-0002$\underline{5672-664 X}$

***** Licenciada en Administración. Alianza de Investigadores Internacionales. E-mail: anarelyspirela@ gmail.com (iD ORCID: https://orcid.org/0000-0001-9251-7055

Doctora en Educación. Docente en el Centro de Bachillerato Tecnológico Agropecuario 197, México. E-mail: irmacastillo197@uemstaycm.gob.mx (iD) ORCID: https://orcid.org/0000-0001-9109-8856

Recibido: 2021-06-14 · Aceptado: 2021-08-31 


\title{
Digital skills and interest in studying in the E-Learning modality in high school students
}

\begin{abstract}
Education is more competitive every day and on the rise towards virtual education in these times of pandemic, which has forced it to be at the forefront in all educational innovation processes. The objective of this study was to evaluate digital skills based on self-perceived knowledge and interest in studying in the e-learning modality of the students of the Centro de Bachillerato Tecnológico Agropecuario 197, Mexico. Quantitative descriptive research was carried out, the sample was made up of 175 individuals, the questionnaire was an adaptation of the Matrix of Digital Skills proposed by the National Autonomous University of Mexico, with 54 questions and it was applied through the Google Applications platform. Among the results found, it stands out that, when self-evaluating their digital skills, students perceive themselves as having an acceptable to very good level, however, they show little interest in studies in e-learning mode due to poor connectivity, low quality of the internet signal, the methodologies and teaching aids, as well as the bad previous experiences. It is concluded that this type of study allows us to glimpse technological literacy strategies that facilitate students to advance towards the knowledge society.
\end{abstract}

Keywords: Digital competences; self-perception; evaluation; digital skills; ICT.

\section{Introducción}

Entre la diversidad de términos que han surgido con el advenimiento de las Tecnologías de Información y Comunicación (TIC) en la educación se encuentra el de competencias digitales. El término emerge a la luz de la sociedad del conocimiento junto a otros conceptos como alfabetización y brecha digital. Ha sido incorporado en documentos que describen políticas oficiales sobre educación, reconociéndose su importancia en la construcción de una sociedad incluyente en la que sus miembros participen de los beneficios del desarrollo de los sistemas de producción de bienes y servicios; y puedan contar con oportunidades equitativas para el trabajo, estudio y en general para la mejora de su condición social (Asociación de Internet. $\mathrm{mx}, 2016)$.

Las TIC contemplan toda forma de tecnología usada para crear, almacenar, intercambiar y procesar información en sus varias formas (Ramírez, 2017). La frecuente interacción/vinculación entre las TIC con los diferentes sectores productivos y gubernamentales ha sido uno de los motivos que han permitido dar pauta para adaptar, así como, promover su uso en la educación (Ramírez y Cruz, 2021). Uno de los retos impostergable de las instituciones educativas es incorporar las TIC en los procesos instruccionales, porque generan experiencias enriquecedoras que de manera creativa y novedosa solventan la ausencia del contacto físico que se vive en el aula tradicional, (Fragozo y Gámez, 2020).

La educación es más competitiva cada día y para mejorar el nivel educativo, es más común la incorporación en los procesos de enseñanza aprendizaje de innovaciones que proporcione a los estudiantes habilidades, competencias y motivaciones que estimulen el aprendizaje significativo y la búsqueda de nuevos conocimientos (Bautista, Martínez e Hiracheta, 2014). Hoy es indudable el reconocimiento estratégico que existe al uso de las tecnologías de la información y comunicación en programas de educación en cualquiera de sus modalidades (Artopoulus y Kozak, 2011).

La llamada educación E-Learning, se denomina como aquel proceso de enseñanzaaprendizaje mediado por las tecnologías de 
la información y la comunicación, el uso de internet, donde existe una separación física entre el estudiantado y los profesores, se caracteriza porque la interacción didáctica puede ser asincrónica o sincrónica, así como en la que el estudiante es el centro del proceso de formación acompañado por tutores y compañeros de clase (Zubieta y Rama, 2015).

Felpeto-Guerrero et al. (2015), señalan que el uso de las TIC en la educación cada día es más común, sin embargo, su implementación no ha sido la mejor, quedando expuesto el gran desafío que ello representa. Leiva y Priegue (2012), destacan la controversia que existe sobre el papel que juegan las tecnologías digitales en los procesos de enseñanzaaprendizaje. Uno de los retos a lograr es que los estudiantes aprendan a emplear las TIC con una actitud crítica y las visualicen como tecnologías que apoyan su formación, aprendizaje y el acceso al conocimiento (Castañeda, 2009). De acuerdo con Hernández (2017), el uso de las TIC en los procesos de enseñanza-aprendizaje requiere, que tanto docentes como estudiantes, desarrollen competencias en el manejo de herramientas tecnológicas que les permita afrontar los retos que significa la educación que se apoya con el uso de las tecnologías digitales.

Para poder contextualizar las características principales de esta modalidad es necesario comprender las dimensiones, significados y alcances que toda propuesta de educación a distancia comparte, el conocimiento y dominio de las mismas es una parte sustantiva para el diseño de Ambientes Virtuales deAprendizaje y materiales didácticos digitales. La flexibilidad es uno de los aspectos más valorados por los estudiantes que deciden optar por la modalidad a distancia. El modelo más flexible en términos de actividades asincrónicas y actividades a distancia, es el modelo interactivo basado en Tecnologías de la Información y Comunicación (TIC), en este modelo se cuenta con un Ambiente Virtual de Aprendizaje (Calzada et al., 2014), tal como se aprecia en el Cuadro 1.

\section{Cuadro 1}

Dimensiones de la educación e-learning

\begin{tabular}{|c|c|c|c|}
\hline Dimensión & Atributo & Significado & Ejemplo \\
\hline \multirow{2}{*}{ Tiempo } & Asíncrono & $\begin{array}{l}\text { La entrega de contenido se produce en un } \\
\text { momento diferente al de la recepción por parte } \\
\text { del estudiante. }\end{array}$ & $\begin{array}{l}\text { Módulo de lectura entregado } \\
\text { por correo electrónico. }\end{array}$ \\
\hline & Síncrono & $\begin{array}{l}\text { La entrega de contenido se produce al mismo } \\
\text { tiempo que la recibe el estudiante. }\end{array}$ & $\begin{array}{l}\text { Proceso de tutoría mediante } \\
\text { una sesión de chat. }\end{array}$ \\
\hline \multirow{2}{*}{ Localización } & $\begin{array}{l}\text { En el mismo } \\
\text { lugar }\end{array}$ & $\begin{array}{l}\text { El estudiante utiliza una aplicación } \\
\text { informática en el mismo lugar físico que otros } \\
\text { estudiantes y / o el docente. }\end{array}$ & $\begin{array}{l}\text { Modificando una hoja de } \\
\text { cálculo de Google Drive } \\
\text { de forma colaborativa en el } \\
\text { salón de clases. }\end{array}$ \\
\hline & Distribuido & $\begin{array}{l}\text { El estudiante utiliza una aplicación informática } \\
\text { en ubicaciones distribuidas, separado de otros } \\
\text { estudiantes y el docente. }\end{array}$ & $\begin{array}{l}\text { Modificando una hoja de } \\
\text { cálculo de Google Drive } \\
\text { de forma colaborativa en } \\
\text { ubicaciones distribuidas. }\end{array}$ \\
\hline
\end{tabular}




\section{Cont... Cuadro 1}

\begin{tabular}{|c|c|c|c|}
\hline \multirow{2}{*}{ Independencia } & Individual & $\begin{array}{l}\text { Los estudiantes trabajan de forma } \\
\text { independiente el uno del otro para completar } \\
\text { las tareas de aprendizaje. }\end{array}$ & $\begin{array}{l}\text { El estudiante realiza } \\
\text { sus consignas de forma } \\
\text { autónoma y es evaluado por } \\
\text { su desempeño individual. }\end{array}$ \\
\hline & Colaborativo & $\begin{array}{l}\text { Los estudiantes trabajan en colaboración con } \\
\text { otros para completar las tareas de aprendizaje. }\end{array}$ & $\begin{array}{l}\text { Los estudiantes participan } \\
\text { en foros de discusión para } \\
\text { compartir ideas. }\end{array}$ \\
\hline \multirow[b]{2}{*}{ Escenario } & $\begin{array}{l}\text { Totalmente } \\
\text { mediado por } \\
\text { TIC }\end{array}$ & $\begin{array}{l}\text { Todo el contenido se entrega a través de } \\
\text { un AVA, no hay contacto físico entre los } \\
\text { participantes. }\end{array}$ & $\begin{array}{l}\text { Una materia disponible en } \\
\text { un AVA para su cursado } \\
\text { totalmente virtual. }\end{array}$ \\
\hline & Combinado & $\begin{array}{l}\text { El AVA se utiliza como un complemento del } \\
\text { aprendizaje tradicional en el aula. }\end{array}$ & $\begin{array}{l}\text { Los materiales didácticos } \\
\text { usados en la clase presencial } \\
\text { están disponibles para su } \\
\text { descarga electrónica por } \\
\text { parte de los alumnos en un } \\
\text { AVA. }\end{array}$ \\
\hline
\end{tabular}

Fuente: Elaboración propia, 2021, adaptado de Calzada et al. (2014).

En este sentido, un Ambiente Virtual de Aprendizaje (AVA) es el espacio intangible creado a través de las TIC donde convergen una serie de elementos (entornos), que permiten el desarrollo del proceso de enseñanzaaprendizaje. Se trata de espacios en donde se crean las condiciones para que el estudiante se apropie de conocimientos, de experiencias, así como elementos que le generen procesos de análisis, reflexión y apropiación; se favorece que por los medios tecnológicos posibilite la mayor interacción del estudiante con el profesor y sus compañeros, las instrucciones y los contenidos están en internet y, se cuenta con un equipo de soporte técnico en línea (Calzada et al., 2014).

De acuerdo con Llomaki et al. (2016), las habilidades digitales son aquellas destrezas, prácticas y competencias que se requieren para el uso de las TIC de manera significativa y como herramienta en cualquier proceso de enseñanza aprendizaje, lo que implica comprender la esencia del fenómeno de tecnologías digitales tanto en la sociedad como en la vida propia, así como la motivación a participar en el mundo digital como actor activo y responsable. Las habilidades digitales pueden definirse como el nivel de dominio que un individuo tiene en el uso de las TIC, lo que requiere conocimiento, practica y experiencia de diferentes tareas relacionadas con ejecuciones de tipo operativo e informacional (Organista et al., 2016).

Para este trabajo, se entiende por competencia digital a la capacidad, conocimiento y habilidades necesarias que demuestra un individuo, para llevar a cabo una tarea de manera satisfactoria cubriendo los requisitos y exigencias que demandan una situación relacionada al proceso de enseñanzaaprendizaje apoyándose en el uso de las tecnologías del aprendizaje y el conocimiento.

Hoy cada día, es más común entre las nuevas generaciones expresiones como nativos digitales e hiperconectados, referencia a personas que conviven con y a través de las llamadas tecnologías de la información y la comunicación (TIC). Se asume la ventaja que conlleva el dominio del uso de las tecnologías por parte de los jóvenes, en la integración de la llamada sociedad digital, y por tanto, en las modalidades de educación no presenciales. Se entiende que, una vez superada la barrera de accesibilidad son capaces de ingresar a una plataforma de enseñanza-aprendizaje, desarrollo y carga de evidencias académicas, además de la colaboración virtual (Avitia y Uriarte, 2017).

Sin embargo, de acuerdo con Gisbert 
y Esteve (2011) en el caso particular de la educación, todavía hoy existe incertidumbre si los estudiantes de bachillerato cuentan con las habilidades y competencias que se requieren para la incorporación exitosa del uso de las tecnologías de la información y la comunicación en procesos de enseñanza aprendizaje, pues no existe consenso sobre cuáles son esas competencias requeridas.

La evaluación de las competencias digitales en diferentes contextos y niveles a través de variadas metodologías, ha mostrado que la autoevaluación es una estrategia útil (Fernández y Torres, 2015; Maderick et al., 2015). Por su parte, Prendes, Gutiérrez y Martínez (2018), advierte que, en el currículum educativo, de manera generalizada, todavía hoy no se toma en cuenta la competencia digital requerida para cursar de manera satisfactoria $\mathrm{y}$ con el mayor provecho que otorga el uso de las TIC en la educación desde el enfoque tecnológico, lo que repercute en el diseño de los Ambientes Virtuales de Aprendizaje.

En este tenor surgen dos preguntas fundamentales: ¿Cuáles son las competencias digitales necesarias? y ¿Cuál es el nivel de dominio que poseen?, que le permitirán continuar sus estudios universitarios teniendo como herramienta el uso de las tecnologías, permitiéndole un mejor desempeño académico. Por lo que, el objetivo del presente estudio fue evaluar las habilidades digitales a partir del conocimiento autopercibido y el interés por estudiar en la modalidad e-learning de los estudiantes de sexto semestre del Centro de Bachillerato Tecnológico Agropecuario 197 en México.

\section{Metodología}

\section{1. Área de estudio}

En la década de 1970 se crearon en México los Centros de Bachillerato Tecnológico Agropecuario (CBTA), como parte de la oferta pública de educación media superior para contribuir a una política educativa fundamental en ese tiempo para el país, la cual privilegiaba la formación de jóvenes para el trabajo por la vía escolar. $\mathrm{Su}$ objetivo es atender la población rural del país, en posibilidad de otorgar el bachillerato formando técnicos medios agropecuarios, que permitan el acceso al trabajo con una elevada calificación y prestigio laboral a través del título de Técnico Medio.

El modelo educativo proponía una educación técnica y modernizadora que desarrollara ejemplos innovadores para las actividades agrícolas, ganaderas, silvícolas, agroindustriales y de servicios relacionados con la agricultura, a los habitantes de las zonas de influencia del plantel. La mayoría de los planteles se ubicaron en zonas rurales de alto rezago educativo y social, cuyos productores utilizan tecnologías tradicionales; aunque también se ubican en regiones con uso de tecnologías intermedias, y en áreas productivas de alta tecnología que orientan su producción a la exportación (De Ibarrola, 2020).

Actualmente, el modelo educativo se basa en el enfoque por competencias (ejemplo de esto se encuentra en artículos de Casanova et al., 2018; Useche y Artigas, 2019; Cejas et al., 2019; Ramos et al., 2021), donde el estudiante participa activamente en la construcción de su conocimiento y en el desarrollo de sus habilidades. La oferta educativa se compone de 31 carreras impartidas en 335 planteles y más de 77 extensiones. Las carreras se agrupan en 1. Sector primario: Producción; 2. Sector secundario: Transformación; y, 3. Sector terciario: Servicios.

El Centro de Bachillerato Tecnológico Agropecuario plantel 197 (CBTA 197) perteneciente a la Unidad de Educación Media Superior Técnica Agropecuaria y Ciencias del Mar (UEMSTAyCM), antes Dirección General de Educación Tecnológica Agropecuaria (DGTA), se ubica en la comisaría de Providencia, municipio de Cajeme, Sonora, México, en el denominado Valle del Yaqui, cuna de la Revolución Verde.

El 15 de febrero de 1989, se abrió como una extensión del CBTA No. 38, con sede en la vecina comisaría Marte R. Gómez 
del mismo municipio. En 1996, reciben la categoría de plantel. Su población estudiantil proviene principalmente de 16 comunidades rurales, así como de la cabecera municipal Ciudad Obregón. El entorno del plantel y de la población en términos socioeconómicos es de media-baja. Las principales actividades económicas más importantes se relacionan con la agricultura, la ganadería, la pesca, así como también la industrial (CBTA 197, 2018).

\subsection{Muestra e instrumentos de colecta de información}

La población total de sexto semestre del plantel es de 500 alumnos. El tamaño de muestra se calculó con un muestreo simple al azar para proporciones, con una confiabilidad del $95 \%$ y una precisión o error de muestro de $5 \%$., utilizando la siguiente fórmula (Creative Research Systems, 2012):

$$
\mathrm{n}=\frac{\mathrm{N} Z^{2}{ }_{\alpha / 2} p_{n} q_{n}}{\mathrm{~N} \mathrm{~d}^{2}+\mathrm{Z}_{\alpha / 2}^{2} p_{n} q_{n}}
$$

Donde:

d Precisión

$Z_{\alpha / 2}$ Confiabilidad

$\mathrm{N}$ Tamaño de la población interés

$p_{n}$ Proporción con la característica de interés

$q_{n}$ Proporción sin la característica de

De una población de 500 estudiantes de sexto semestre del CBTA 197, se le aplicó un cuestionario a una muestra representativa de 175 estudiantes, misma que se calculó de la siguiente manera: $\mathrm{N}=500 ; \mathrm{z}_{\mathrm{x} / 2}=1.64 ; \mathrm{d}$ $=0.75 ; \mathrm{p}_{\mathrm{n}}=0.25 ; \mathrm{y}, \mathrm{q}_{\mathrm{n}}=0.25$. Se calculó el tamaño de muestra con el supuesto de varianza máxima: $\mathrm{p}=0.25$ y $\mathrm{q}=0.25$. El tamaño de muestra fue de: $\mathrm{n}=175$ estudiantes.

Con este tamaño de muestra definido se procedió a levantar la encuesta en los estudiantes del sexto semestre del bachillerato, seleccionando en forma aleatoria a los alumnos que fueron entrevistados; esta encuesta se realizó a través de la plataforma Aplicaciones de Google, utilizando la herramienta Formularios y distribuyéndose vía WhatsApp.

El instrumento empleado es una modificación de la matriz de habilidades digitales propuesta por la Dirección General de Cómputo y de Tecnologías de la Información y Comunicación (DGTIC) de la Universidad Nacional Autónoma de México (UNAM, 2014). El cuestionario utilizado consta de cuatro secciones: 1) Datos generales; 2) Acceso a internet; 3) Habilidades digitales; y, 4) Interés y experiencia en estudios en línea. Para el análisis, en la primera sección, se consideraron tres preguntas cerradas sobre datos generales; respecto a la sección acceso a internet, fueron ocho preguntas; mientras que, en habilidades digitales, consistió en 36 preguntas cerradas categorizadas con afirmaciones y donde las respuestas se evalúan a través de una escala de Likert de cinco puntos: Muy poco, Poco, Regular, Parcialmente, Totalmente. La cuarta sección, contempló ocho preguntas.

Para la integración del cuestionario, este se dividió en diez secciones, donde el de habilidades digitales consideró seis apartados. En total se emplearon 54 reactivos, tal como se muestra a continuación en la Tabla 1 . 
Ramírez García, Adán Guillermo; Rodríguez Sauceda, Elvia Nereyda; Pirela Hernández, Ana Arelis y Castillo Escalante, Irma Cecilia

Habilidades digitales e interés por estudiar en la modalidad E-Learning en estudiantes de Bachillerato

\section{Tabla 1}

Relación de reactivos empleados por categoría.

\begin{tabular}{ll}
\hline Categoría & Reactivos \\
\hline
\end{tabular}

1) Datos generales

2) Acceso a internet

3) Habilidades digitales

3.1) Comunicación y colaboración

3.2) Ambientes virtuales

3.6) Uso de hardware

Fuente: Elaboración propia, 2021.

\subsection{Estadísticos descriptivos: Media, desviación estándar y número de participantes}

Los métodos de la Estadística Descriptiva o Análisis Exploratorio de Datos, ayudaron a presentar los datos de modo tal que sobresalga su estructura. Se trató con este análisis, detectar tanto las características sobresalientes como las características inesperadas. La media general de elementos se encuentra en 3.157 y una varianza de 5.82. Las correlaciones se encuentran en un rango de entre -0.92 a 0.77 . La covarianza se encuentra entre valores de -1.5771 y 2.305 , tal como se puede evidenciar en la Tabla 2. 
Tabla 2

Estadísticos descriptivos: Media, desviación estándar y número de participantes

\begin{tabular}{lccccccc}
\hline & Media & Mínimo & Máximo & Rango & $\begin{array}{c}\text { Máximo/ } \\
\text { Mínimo }\end{array}$ & $\begin{array}{c}\text { Varianza } \\
\text { elementos }\end{array}$ \\
\hline Medias de elemento & 3.157 & 1.333 & 18.388 & 17.055 & 13.791 & 5.822 & 54 \\
Varianzas de elemento & 3.174 & 0.098 & 75.038 & 74.940 & 767.398 & 108.773 & 54 \\
Covarianzas entre elementos & 0.287 & -1.571 & 2.305 & 3.875 & -1.467 & 0.145 & 54 \\
Correlaciones entre elementos & 0.151 & -0.925 & 0.773 & 1.699 & -0.836 & 0.052 & 54 \\
\hline
\end{tabular}

Fuente: Elaboración propia (2021).

De acuerdo con Arias (2008), los datos deben estar libres de multicolinealidad, la cual se presenta cuando variables diferentes miden el mismo constructo, lo que implica una correlación muy elevada entre ellas $(0.90$ o superior). En este estudio, se obtuvo una correlación media inter-item de 0.151 y al revisar la matriz de correlaciones en ningún caso se presentaron valores de correlación superiores a 0.90 . De igual manera, la media de covarianzas entre elementos es de 0.287 , lo que demuestra que los datos están libres de multicolinealidad.

\subsection{Cálculo del Alfa de Cronbach}

El Alfa de Cronbach es un coeficiente que se utiliza para conocer la fiabilidad de una escala o test. En este caso, la fiabilidad se entiende como la ausencia de errores de medida en un test y la precisión de su medición. Respecto a la consistencia interna, se refiere al grado en que todos los items del test covarian entre sí (Oviedo y Campo-Arias, 2005). Para el cálculo del alfa de Cronbach se emplearon las siguientes variantes:

a. El Alfa de Cronbach: Indica la confiabilidad del instrumento. Se calculó a partir de las varianzas utilizando la siguiente fórmula:

$$
\propto=\frac{K}{K-1}\left\lfloor 1-\frac{\sum V i}{V t}\right\rfloor
$$

\section{Dónde:}

$$
\begin{aligned}
& \propto=\text { Alfa de Crombach } \\
& K=\text { Número de Items } \\
& V_{i}=\text { Varianza de cada Item } \\
& V_{t}=\text { Varianza del total }
\end{aligned}
$$

De igual manera, para al análisis del coeficiente del alfa de Cronbach se usó como referencia lo indicado en el siguiente Cuadro 2. 
Habilidades digitales e interés por estudiar en la modalidad E-Learning en estudiantes de

Bachillerato

\section{Cuadro 2}

\section{Criterios de interpretación del} coeficiente de Alfa de Crobach

\begin{tabular}{cl}
\hline Valores de Alfa & \multicolumn{1}{c}{ Interpretación } \\
\hline $0.90-1.00$ & Se califica como muy satisfactoria \\
$0.80-0.89$ & Se califica como muy adecuada \\
$0.70-0.79$ & Se califica como moderada \\
$0.60-0.69$ & Se califica como baja \\
$0.50-0.59$ & Se califica como muy baja \\
$\leq 0.50$ & Se califica como no confiable \\
\hline
\end{tabular}

Fuente: Elaboración propia (2021).

b. Matriz de correlaciones interelementos: Muestra las correlaciones entre los items. Empleando el paquete estadístico SPSS se calculó las correlaciones productomomento de Pearson; como su procedimiento de estimación por defecto. El Alfa de Cronbach basada en elementos tipificados se estimó a partir de las correlaciones. Igualmente se obtuvo el coeficiente alfa ordinal mediante la estimación de la matriz de correlaciones policóricas.

c. Matriz de covarianzas: Muestra las correlaciones entre los items. El análisis de covarianza se utilizó como una prueba Paramétrica. Lo que permitió analizar los factores dentro de una población. Además, permitió cuantificar hasta qué punto dos variables son independientes. La covarianza entre dos variables $\mathrm{S}_{\mathrm{xy}}$, indicó si la posible relación entre dos variables es directa o inversa. Como a continuación se indica: Directa: $\mathrm{S}_{\mathrm{xy}}$ $>0$; Inversa: $\mathrm{S}_{x y}<0$; e, Incorreladas: $\mathrm{S}_{\mathrm{xy}}=0$. Para obtener las covarianzas se calcularon con la siguiente fórmula:

$$
S_{x y}=\frac{1}{n} \sum_{i}\left(x_{i}-\bar{x}\right)\left(y_{i}-\bar{y}\right)
$$

d. Nivel de habilidad: El nivel de habilidad se estimó con base en la escala ordinal propuesta por Carrera, Vaquero y Balsells (2011), con las siguientes opciones de autopercepción: >4 Muy buena; 3.0 a 3.9 Buena; 2.0 a 2.9 Regular; 1.0 a 1.9 Mala; y, $<1$ Muy Mala.

\subsection{Adecuación de la base de datos}

Se realizaron ajustes con la finalidad de identificar errores y datos atípicos. Primeramente, se hizo una revisión de las escalas de medición de las variables para verificar que coincidieran con las previamente establecidas.

\subsection{Análisis de fiabilidad de las puntaciones}

Los resultados para el cálculo del coeficiente alfa de Cronbach se detallan a continuación en la Tabla 3. Los hallazgos muestran una alta consistencia interna del cuestionario aplicado (coeficiente alfa de Cronbach $=0.838$ ). De acuerdo con este índice, un instrumento es consistente si sus valores oscilan entre 0.70 y 0.90 ; valores menores a 0.70 sugieren que la escala es poco homogénea y mayores a 0.90 pueden indicar la existencia de items redundantes (Panayides, 2013).

Tabla 3

Relación de reactivos empleados por categoría

\begin{tabular}{ccccccc}
\hline $\begin{array}{c}\text { Grupos de } \\
\text { variables }\end{array}$ & $\begin{array}{c}\text { Alfa de Cronbach } \\
\text { (Varianza de los } \\
\text { ítems) }\end{array}$ & $\begin{array}{c}\text { Alfa de } \\
\text { Cronbach } \\
\text { basada en } \\
\text { elementos } \\
\text { estandarizados } \\
\text { (SPSS) }\end{array}$ & $\begin{array}{c}\text { N de } \\
\text { elementos }\end{array}$ & $\begin{array}{c}\text { Desviación } \\
\text { Asimetría }\end{array}$ & $\begin{array}{c}\text { Cursosis } \\
\text { centrada } \\
\text { en 0 }\end{array}$ \\
\hline Todas las variables & 0.838 & 0.903 & 54 & 30.450 & -0.883 & 0.765
\end{tabular}




\section{Cont... Tabla 3}

\begin{tabular}{lcccccc}
$\begin{array}{l}\text { Habilidades } \\
\text { digitales }\end{array}$ & 0.839 & 0.839 & 8 & 7.25 & -0.380 & -0.27 \\
$\begin{array}{l}\text { Comunicación y } \\
\text { colaboración }\end{array}$ & 0.838 & 0.840 & 8 & 7.49 & -0.339 & -0.706 \\
$\begin{array}{l}\text { Ambientes } \\
\text { virtuales }\end{array}$ & 0.803 & 0.806 & 4 & 3.88 & -3.499 & 11.287 \\
$\begin{array}{l}\text { Administración de } \\
\text { la información }\end{array}$ & 0.887 & 0.887 & 6 & 5.79 & -0.433 & -0.612 \\
$\begin{array}{l}\text { Seguridad de la } \\
\text { información }\end{array}$ & 0.695 & 0.695 & 4 & 3.78 & 0.339 & -0.405 \\
Manejo de medios & 0.797 & 0.796 & 3 & 2.98 & -1.432 & 1.139 \\
\hline
\end{tabular}

Fuente: Elaboración propia, 2021.

Como se observa, en los coeficientes de cada dimensión se obtuvieron valores superiores al 0.70 y en el global 0.80 . Solamente el grupo de variables para Seguridad de la información presenta valores inferiores a 0.70 , lo que puede sospechar de Ítems redundantes. Por lo tanto, las demás puntuaciones poseen un buen nivel de fiabilidad.

Se omitieron las categorías datos generales, uso de hardware, acceso a internet e interés y experiencia en estudios en línea, debido a que el coeficiente no es apropiado para una escala que explora un solo factor, siendo tres el número mínimo de Ítems o preguntas con más de 20 respuestas (Panayides, 2013). Para uso de hardware, los bajos valores se debieron a la dicotomía de las respuestas y para acceso a internet e interés y experiencia en estudios en línea fue por más de 20 respuestas. Sin embargo, se consideran en los resultados del estudio por la información que aportan.

De estos valores se observa que para el caso de la escala de Likert de 8 puntos se tendría calculando la varianza de los items un valor de 0.838 , que se encontraría dentro del límite de 0.80 a 0.89 que indica una buena consistencia interna para esta escala. El cálculo del alfa de Cronbach estandarizada se encuentra en el intervalo más alto que se califica con una consistencia interna como muy satisfactoria. Este último valor de 0.90 se explica pues se está utilizando el método de cálculo con matrices de correlación de Pearson.

Es conveniente señalar que la fiabilidad comprende dos componentes, la consistencia interna, que refiere al grado en que los distintos items miden un mismo constructo, a lo que en Organista et al. (2016), fueron reportadas las evidencias de validez del cuestionario. El otro componente, de la estabilidad temporal, implica que el instrumento arroje el mismo resultado ante diversas aplicaciones a un objeto de estudio que se espera permanezca invariable. En este sentido, no se cuenta con datos de una segunda aplicación que permita explorar la estabilidad temporal.

También se calculó el número de dimensiones a considerar implementando el Análisis Paralelo. En este caso, Likert_8, el valor obtenido fue 1 de dimensión. La correlación policórica se aconseja cuando la distribución univariada de los items ordinales es asimétrica o tiene exceso de curtosis. $\mathrm{Si}$ ambos índices tienen valores absolutos menores de 1 se recomienda la correlación de Pearson.

\section{Resultados y discusión}

Se presentan los resultados en el siguiente orden: Datos generales, acceso a internet, habilidades digitales (comunicación y colaboración, ambientes virtuales, administración de la información, seguridad 
Ramírez García, Adán Guillermo; Rodríguez Sauceda, Elvia Nereyda; Pirela Hernández, Ana Arelis y Castillo Escalante, Irma Cecilia

Habilidades digitales e interés por estudiar en la modalidad E-Learning en estudiantes de Bachillerato

de la información, manejo de medios y uso de hardware), finalizando con el interés y experiencia por estudios en línea.

\subsection{Descripción de los participantes (Datos generales)}

Los 175 entrevistados pertenecen al CBTA 197, con edades en promedio de 18 años, 63 fueron hombres (36\%) y 112 mujeres (64\%), tal como se muestra en el Gráfico I.

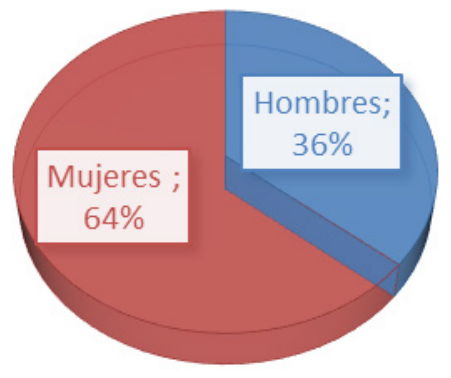

Fuente: Elaboración propia, 2021.

\section{Gráfico I: Sexo de los estudiantes entrevistado}

\subsection{Acceso a internet}

Respecto a si tienen acceso a internet en casa, el $66 \%$ de los entrevistados indican que sí. El 27\% (48) manifiesta que el dispositivo que utilizan para navegar en internet es a través de la PC, 65\% (113) sostiene que lo hace a través de un Smartphone, y el $8 \%$ restante lo hace con una laptop o una Tablet en igual número (7\%). Asimismo, el $83 \%$ de los encuestados diariamente se conecta entre cuatro y cinco horas al Internet.

El proveedor de internet que más utilizan es Megacable (42\%), seguido por Telcel (35\%) e Infinitum (17\%), el 6\% restante cuentan con otro servicio. Respecto a la velocidad de internet contratada solo el $44 \%$ sabe cuál es. El lugar habitual donde accede a internet es la casa (76\%), la escuela $14 \%$ y en un cibercafé $10 \%$.

Sobre las principales redes sociales utilizadas destacan: Facebook (99\%), WhatsApp (88\%), Instagram (71\%), y Tiktok (40\%), Snapchat (36\%), y Twitter (23\%). El $26 \%$ manifiesta haber hecho al menos una compra en internet, mientras que la gran mayoría (74\%) nunca ha utilizado el internet para comprar algo. La principal plataforma para compras es Mercado Libre (14\%).

Contrastando con resultados anteriores, se observa que de acuerdo con la Asociación de Internet.mx (2016; 2017), el 65\% se conecta diariamente entre tres y cuatro horas al Internet. Su conexión es desde el hogar (54\%) y los dispositivos de conexión más utilizados sobresalen los Smartphone 90\%, Laptop 73\%, Tablet $52 \%$, PC $42 \%$. En cuanto a los hábitos de conexión el $82 \%$ tiene una conexión WIFI contratada, el $61 \%$ un plan de datos y un $26 \%$ la conexión WIFI que utiliza es de acceso público. El lugar más frecuente de conexión es el hogar $82 \%$, en cualquier lugar el $74 \%$, el trabajo $60 \%$, la escuela $10 \%$, lugares públicos $19 \%$ seguido de los llamados Cibercafe 5\%, mostrando semejanzas con los resultados del presente estudio.

Las redes sociales más utilizadas en los estudios de la Asociación de Internet.mx (2016; 2017) son Facebook (95\%), WhatsApp (93\%), YouTube (72\%), Twitter (66\%), Instagram (59\%). Cinco redes sociales en promedio poseen cada usuario en México, mientras que el $1 \%$ manifiesta no estar inscrito en ninguna. En cuanto a la categoría acceso a internet, comparando los resultados de esta investigación con los de la Asociación, arrojan similitudes, entre las que destacan, las evidenciadas en la Tabla 4. 
Tabla 4

Similitudes en la categoría acceso a internet

\begin{tabular}{lcc}
\hline \multicolumn{1}{c}{ Ítem } & \% Obtenido & $\begin{array}{c}\text { \% } \\
\text { Otros estudios }\end{array}$ \\
\hline Disponibilidad de conexión a internet en el hogar & 66 & 82 \\
El lugar más frecuente de conexión & 76 & 82 \\
Smartphone como mayor dispositivo de conexión & 65 & 90 \\
Principal actividad online redes sociales & 100 & 83 \\
\hline
\end{tabular}

Fuente: Elaboración propia, 2021, adaptado de Asociación de Internet.mx (2016; 2017).

\subsection{Habilidades digitales}

En la Tabla 5, se muestra el concentrado de resultados de todos los aspectos evaluados. Los valores medios obtenidos se ubicaron en el rango 3.74-2.10, de la escala 1-4 considerada.
El valor medio mayor le correspondió a la categoría de habilidad para acceso a la información de interés, mientras que el valor medio mínimo (2.1086) fue si sabían identificar metabuscadores. Es de notar la cercanía entre los cuatro valores medios obtenidos.

Tabla 5

Habilidades digitales

\begin{tabular}{|c|c|c|c|}
\hline ACCESO A LA INTERNET & Media & D.E. & $\begin{array}{c}\text { Nivel de } \\
\text { habilidad }\end{array}$ \\
\hline ¿Qué tan hábil eres para accesar a información de tu interés? & 3.7486 & 1.03095 & 3 \\
\hline $\begin{array}{l}\text { ¿Cuándo buscas información en Internet principalmente usas } \\
\text { sitios institucionales como fuente? }\end{array}$ & 3.4857 & 1.03311 & 3 \\
\hline $\begin{array}{l}\text { Eres capaz de realizar trámites administrativos en línea como } \\
\text { solicitud de constancias de inscripción, CURP, Seguro social, } \\
\text { etc. }\end{array}$ & 3.1943 & 1.29853 & 3 \\
\hline ¿Empleas bibliotecas digitales en línea? & 2.3543 & 1.24576 & 2 \\
\hline $\begin{array}{l}\text { ¿Sabes cómo citar en un documento las fuentes de internet } \\
\text { empleadas? }\end{array}$ & 3.2114 & 1.26668 & 3 \\
\hline ¿Organizas tus sitios favoritos de internet en carpetas? & 2.6857 & 1.39316 & 2 \\
\hline $\begin{array}{l}\text { ¿Sabes cómo personalizar la barra de herramientas del } \\
\text { navegado? }\end{array}$ & 3.36 & 1.41486 & 3 \\
\hline ¿Utilizas operadores booleanos para hacer búsquedas especiales? & 2.12 & 1.02967 & 2 \\
\hline ¿Sabes identificar metabuscadores? & 2.1086 & 1.16692 & 2 \\
\hline COMUNICACIÓN Y COLABORACIÓN & Media & D.E. & $\begin{array}{l}\text { Nivel de } \\
\text { habilidad }\end{array}$ \\
\hline $\begin{array}{l}\text { ¿Públicas y compartes archivos (audio, vídeo, imagen y } \\
\text { documentos) en redes sociales (Faceboock, Twitter, Pinterest)? }\end{array}$ & 3.7953 & 1.37164 & 3 \\
\hline Utilizas chats o video chat (Hangouts, Skype, WhatsApp) & 3.7485 & 1.37239 & 3 \\
\hline $\begin{array}{l}\text { ¿Participas en grupos (Grupo de Facebook, Grupo de Google, } \\
\text { Yahoo Groups, Comunidad Google +, etc)? }\end{array}$ & 3.3158 & 1.44497 & 3 \\
\hline ¿Sabes crear, abrir y eliminar archivos compartidos? & 4.0468 & 1.12623 & 4 \\
\hline $\begin{array}{l}\text { ¿Sabes instalar antivirus para Android, Windows, IOS en un } \\
\text { dispositivo móvil? }\end{array}$ & 2.8129 & 1.42666 & 2 \\
\hline
\end{tabular}




\section{Cont... Tabla 5}

¿Sabes gestionar carpetas o etiquetas de correo electrónico?

¿Sabes sincronizar dos o más cuentas de correo electrónico?

Eres capaz de editar, compartir y descargar documentos en la nube.

$\begin{array}{lll}2.8304 & 1.30177 & 2 \\ 2.9825 & 1.41202 & 2 \\ 2.9942 & 1.46125 & 2\end{array}$

\begin{tabular}{|c|c|c|c|}
\hline AMBIENTES VIRTUALES & Media & D.E & $\begin{array}{l}\text { Nivel de } \\
\text { habilidad }\end{array}$ \\
\hline $\begin{array}{l}\text { ¿Sabes cómo enviar tareas y revisar los comentarios del docente } \\
\text { en una plataforma educativa? }\end{array}$ & 3.3468 & 1.3056 & 3 \\
\hline ¿Puedes localizar, desglosar y utilizar recursos y materiales? & 3.3121 & 1.19384 & 3 \\
\hline ¿Puedes participar en wikis dentro de una plataforma educativa? & 2.474 & 1.17417 & 2 \\
\hline $\begin{array}{l}\text { ¿Sabes cómo participar en un foro (colocar un nuevo tema de } \\
\text { discusión, ordenar respuestas?? }\end{array}$ & 2.6936 & 1.21225 & 2 \\
\hline ADMINISTRACIÓN DE LA INFORMACIÓN & Media & D.E. & $\begin{array}{l}\text { Nivel de } \\
\text { habilidad }\end{array}$ \\
\hline $\begin{array}{l}\text { ¿Puedes trabajar con documentos en procesadores de texto } \\
\text { (crear, abrir, guardar y editar documentos)? }\end{array}$ & 3.7588 & 1.18449 & 3 \\
\hline $\begin{array}{l}\text { ¿Puedes trabajar con hojas de cálculo (crear abrir o editar libros } \\
\text { y hojas, nombrar, editar, insertar, ocultar y mover)? }\end{array}$ & 3.1176 & 1.20071 & 3 \\
\hline $\begin{array}{l}\text { ¿Te considero eficiente para crear bases de datos con un gestor } \\
\text { de base de datos? }\end{array}$ & 2.6059 & 1.1163 & 2 \\
\hline $\begin{array}{l}\text { ¿Eres capaz de paginar un documento con secciones en un } \\
\text { procesador de texto? }\end{array}$ & 2.7471 & 1.20188 & 2 \\
\hline ¿Eres capaz de crear y editar macros en una hoja de cálculo? & 2.5 & 1.23197 & 2 \\
\hline $\begin{array}{l}\text { ¿Sabes crear tablas dinámicas de contenido e índices en un } \\
\text { procesador de texto? }\end{array}$ & 2.8059 & 1.29312 & 2 \\
\hline SEGURIDAD DE LA INFORMACIÓN & Media & D.E. & $\begin{array}{l}\text { Nivel de } \\
\text { habilidad }\end{array}$ \\
\hline $\begin{array}{l}\text { ¿Tienes la costumbre de cerrar sesiones (redes sociales, correo } \\
\text { electrónico, blackboard), sobre todo en equipos públicos? }\end{array}$ & 4.3198 & 1.1119 & 4 \\
\hline ¿Borras historial y cookies al emplear equipo ajeno? & 3.3953 & 1.43294 & 3 \\
\hline $\begin{array}{l}\text { ¿Sabes identificar las diferentes formas de robo de datos: } \\
\text { spyware, malware, registro de captura de teclas? }\end{array}$ & 2.7267 & 1.3511 & 2 \\
\hline $\begin{array}{l}\text { ¿Periódicamente haces respaldos de seguridad de la } \\
\text { información? }\end{array}$ & 3.1163 & 1.31495 & 3 \\
\hline MANEJO DE MEDIOS & Media & D.E & $\begin{array}{l}\text { Nivel de } \\
\text { habilidad }\end{array}$ \\
\hline Cito las fuentes de donde se extrae las imágenes que utilizo & 2.6286 & 1.07441 & 2 \\
\hline $\begin{array}{l}\text { Soy capaz de integrar una secuencia de imágenes para crear una } \\
\text { animación. }\end{array}$ & 2.5486 & 1.20186 & 2 \\
\hline $\begin{array}{l}\text { Hago uso de software para conversación, creación y edición de } \\
\text { audio. }\end{array}$ & 2.56 & 1.24826 & 2 \\
\hline USO DE HARDWARE & Media & D.E & $\begin{array}{l}\text { Nivel de } \\
\text { habilidad }\end{array}$ \\
\hline $\begin{array}{l}\text { ¿Sabes instalar y configurar dispositivos periféricos inalámbricos } \\
\text { y alámbricos, multifuncionales, impresoras, teclados, cámaras } \\
\text { web, etcétera? } \\
\text { ¿Te consideras eficiente para instalar un sistema operativo en mi } \\
\text { equipo de cómputo? }\end{array}$ & 1.486 & 1.486 & 1 \\
\hline
\end{tabular}

Nota: $>4$ Muy buena; 3.0 a 3.9 Buena; 2.0 a 2.9 Regular; 1.0 a 1.9 Mala y, $<1$ Muy Mala

Fuente: Elaboración propia, 2021. 
De acuerdo con la escala de autopercepción, se obtuvo una media general de 3.78, Nivel 3 (bueno) indicativo que los estudiantes estarían en condiciones de realizar las tareas señaladas sin ayuda. Respecto a los puntajes medios alcanzados por encuesta, el valor mínimo fue 1.5 y el máximo 4; además, se obtuvo $1.7 \%$ de valores perdidos.

\section{a. Comunicación y colaboración}

En la categoría de comunicación y colaboración, existe una amplia participación de los estudiantes en redes sociales, así como una autosuficiencia percibida para actividades de colaboración en línea. Es importante señalar que se consideran muy buenos para el manejo de archivos en internet como abrir, crear y eliminar archivos compartidos. También respecto a este rubro de comunicación y colaboración, se tiene una percepción buena, así como muy buena con valores que oscilan entre 4.04 a 2.98 .

\section{b. Ambientes virtuales}

De igual manera se consideran competentes para participar en actividades básicas dentro de los ambientes virtuales. Según la escala planteada de autopercepción esta es de buena a mala regular, y se auto perciben como regulares para participar en wikis en plataformas educativas, así como participar en foros.

\section{c. Administración de la información}

En la administración de la información, necesaria para el desarrollo de actividades académicas de procesamiento, existe dispersión en la calificación que se otorgan los estudiantes, encontrándose mayor confianza en su manejo de procesadores de texto por encima de las bases de datos. Considerándose buenos y regulares, respetivamente.

\section{d. Seguridad de la información}

La seguridad de la información, es otro tema que ha sido señalado como primordial para el desarrollo de una ciudadanía digital y parte de la competencia digital esperada. En esta categoría los estudiantes evaluados se perciben con un nivel medio de habilidad digital, estando habituados a realizar la acción básica de seguridad informática de cerrar sesiones y borrar historiales en los equipos de cómputo. Con valores de autopercepción de muy buenos en el caso de la costumbre de cerrar sesiones (redes sociales, correo electrónico, blackboard), sobre todo en equipos públicos. Manifestando una mala autopercepción en la identificación de formas de robo de datos.

\section{e. Manejo de medios y hardware}

Por su parte, en el manejo de medios y hardware existe una capacidad auto percibida de mala a muy mala, reconociéndose los estudiantes como malos de instalar hardware o software, pero con mejor capacidad para emplear software especializado para edición de imágenes o creación de secuencias de animación.

En un estudio similar realizado por Avitia y Uriarte (2017), encontraron que, la mayoría de los estudiantes que respondieron la encuesta cuenta con un nivel 2 o medio de habilidades, al considerarse capaces de realizar actividades asociadas a la búsqueda, organización y valoración de la información disponible en la red, no así para emplear búsquedas avanzadas a través de operadores booleanos o metabuscadores.

En cuanto a la Comunicación y Colaboración, existe una amplia participación de los estudiantes en redes sociales, así como una autoeficacia percibida para actividades de colaboración en línea. En la Administración de la Información (empleo de procesadores de texto, hojas de cálculo, bases de datos) necesarios para el desarrollo de actividades académicas de procesamiento, existe dispersión en la calificación que se otorgan los 
estudiantes encontrándose mayor confianza en su manejo de procesadores de texto por encima de las bases de datos.

La Seguridad de la Información, es otro tema que ha sido distinguido como fundamental en el desarrollo de una competencia digital. En este rubro los estudiantes evaluados en el estudio de Avitia y Uriarte (2017), se perciben con un nivel medio de habilidad digital estando habituados a realizar la acción básica de seguridad informática de cerrar sesiones y borrar historiales en los equipos de cómputo. Por su parte, en el Manejo de Medios y Hardware existe una capacidad auto percibida de nivel medio, reconociéndose los estudiantes como capaces de instalar hardware o software, pero capaces en menor medida de emplear software especializado para edición de imágenes o creación de secuencias de animación, contrastando en este aspecto con los resultados del presente estudio donde los estudiantes se perciben con poca o ninguna capacidad al respecto.

\subsection{Interés y experiencia en estudios en línea}

Respecto al interés y experiencia en estudios en línea, el $39 \%$ manifiesta que alguna vez ha tomado al menos un curso en línea. De ellos, el 30\% manifiesta que su experiencia fue de regular a buena; mientras que el $70 \%$ restante dice que fue mala o muy mala. La opción de continuar sus estudios en la modalidad en línea, el 21\% lo considera como una opción viable, mientras que el 51\% no lo considera y el $37 \%$ está indeciso.

El $46 \%$ indica que todavía no sabe qué va a estudiar egresando del bachillerato. El interés por estudios relacionados a medicina y enfermería es de 14\%, llama la atención que las ciencias agropecuarias solo tiene el interés por un 5\% de la población en estudio. Las ciencias económico-administrativas tienen el $12 \%$, informática y computación $8 \%$, y los temas relacionados con la educación y la psicología $9 \%$, mientras que los estudios relacionados a la ingeniería son de $6 \%$.

El $89 \%$ de la muestra, manifiesta que no sabe nada de la Universidad Autónoma Chapingo. Los que conocen a la Universidad, saben que ahí se puede estudiar Agronomía e Ingeniería Agroindustrial. El 11\% sí considera la opción de estudiar en Chapingo, el $47 \%$ no muestra interés alguno, y el $42 \%$ está abierto a recibir información de la Universidad para contemplarla como opción de estudiar en Chapingo.

De acuerdo con la Asociación de Internet.mx (2016), las actividades online y las redes sociales permanecen como la principal actividad en línea (83\%), seguido de enviar y recibir correo electrónico $(78 \%)$, enviar/recibir mensajes instantáneos (chats)/llamadas (77\%), y búsqueda de información (74\%); mientras que estudiar en línea solo representa el $38 \%$ seguido por compras en línea $37 \%$.

En resumen, el nivel general de habilidad mostrado por los estudiantes participantes en este estudio va de buena a regular, conglomerando un número importante de individuos que auto perciben que tienen habilidades digitales según lo muestra el Gráfico II. Y es por ello, que es importante que los docentes fomenten su adquisición, puesto que, de acuerdo con Fajardo, Villata y Salmerón (2016), estas habilidades deben fomentarse en especial en los currículos de las instituciones, de manera tal que, el profesorado tenga directrices precisas de cómo construir conocimientos utilizando las herramientas digitales para guiar a sus estudiantes. 


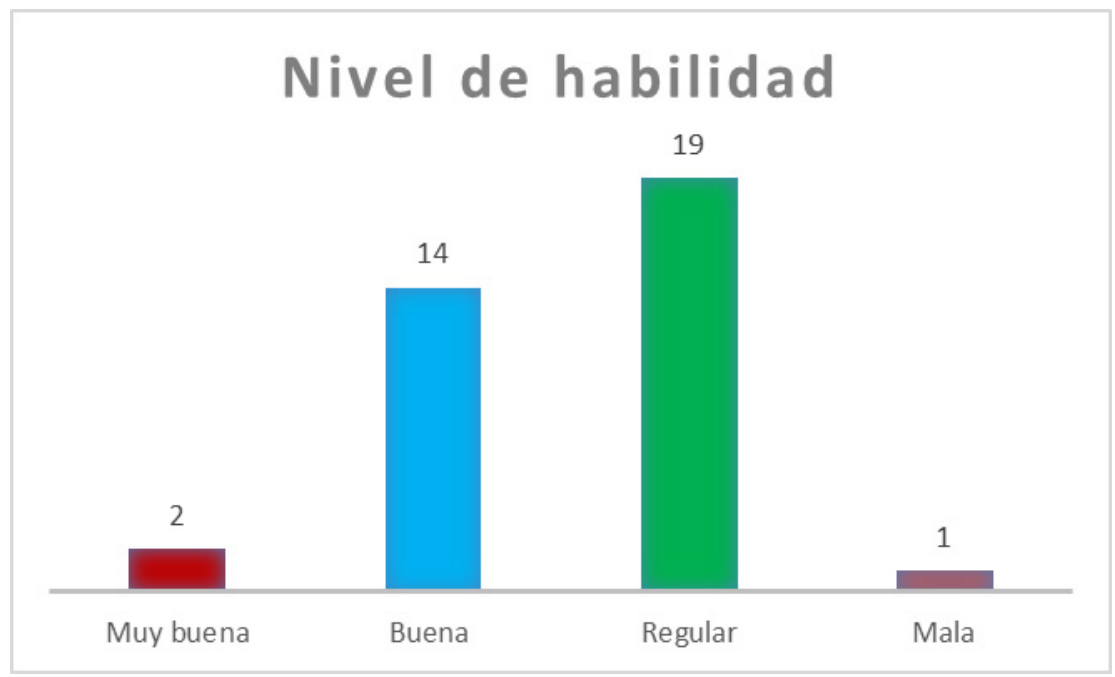

Fuente: Elaboración propia, 2021.

\section{Gráfico II: Nivel de habilidad auto percibida}

Al observar la gráfica, se puede detallar que un gran número de estudiantes autoperciben que tiene habilidades digitales con las cuales pueden defenderse en un mundo altamente digitalizado, y que son un elemento importante en la generación de conocimiento, puesto que como sostiene Gallardo (2012), es erróneo pensar que los estudiantes no requieren una enseñanza formal de estas habilidades; y que como manifiestan Avita y Uriarte (2017), las mismas les serán utilies para acceder y participar en programas académicos en modalidades en línea o e-learning, modalidad que día a día está ganando más adeptos por sus beneficios.

\section{Conclusiones}

Por lo anteriormente descrito, se concluye que los individuos en estudio al auto evaluarse se perciben con un nivel de aceptable a muy bueno respecto a sus habilidades digitales. Sin embargo, muestran poco interés por continuar sus estudios superiores en la modalidad e-learning, debido a que, en su experiencia, hay una deficiente conectividad, baja calidad de la señal de internet y que las metodologías de enseñanza y los medios de instrucción, no se comparan con la educación presencial. Se infiere que por la edad promedio de la muestra analizada, tienen mucha familiaridad con el uso de la tecnología y las habilidades necesarias para su uso, y que el acceso a internet tiene fines recreativos, por lo que muestran resistencia para usarlo como llave de acceso a la educación.

Dada la nueva normalidad causada por la pandemia del COVID-19 y el inminente crecimiento de la oferta de educación en modalidad a distancia, el presente estudio proporciona información que logra evidenciar la importancia que tiene el nivel bachillerato, para desarrollar ambientes virtuales innovadores que fomenten el autoaprendizaje y que sean compatibles en smartphones, pues el uso de computadoras de escritorio y tabletas cada día es menor, con el fin que cada vez 
Ramírez García, Adán Guillermo; Rodríguez Sauceda, Elvia Nereyda; Pirela Hernández, Ana Arelis y Castillo Escalante, Irma Cecilia

Habilidades digitales e interés por estudiar en la modalidad E-Learning en estudiantes de Bachillerato

más estudiantes consideren la posibilidad de continuar sus estudios superiores, de capacitación y/o actualización en esta modalidad.

Respecto a la metodología empleada en este estudio, se considera que es adecuada, valida y replicable. Sin embargo, por lo reducido de la muestra el alcance de los hallazgos aquí presentados es limitante, que invitan a continuar con estudios similares en diferentes niveles educativos, extractos sociales y otros sistemas formativos, que arrojen información que permita la toma de decisiones, así como propuestas de políticas públicas respecto al uso de las tecnologías de la información y la comunicación en el rubro de la educación.

Finalmente, este tipo de estudios permite vislumbrar estrategias de alfabetización tecnológica para reducir la brecha digital e implementar acciones en los planteles educativos que faciliten a los estudiantes el dominio de entornos digitales que les permita avanzar hacia la denominada sociedad del conocimiento.

\section{Referencias bibliográficas}

Arias, B. (2008). Desarrollo de un ejemplo de análisis factorial confirmatorio con LISREL, AMOS y SAS. En M. Á. Verdugo, M. Crespo, M. Badía, y B. Arias (Eds.), Metodología en la investigación sobre discapacidad. Introducción al uso de las ecuaciones estructurales. VI Seminario Cientifico SAID (pp. 75-120). Publicaciones INICO.

Artopoulus, A., y Kozak, D. (2011). Tsunami 1:1: Estilos de adopción de tecnología en la educación latinoamericana. Revista Iberoamericana de Ciencia, Tecnología y Sociedad, 6(18),137-171.

Asociación de Internet.mx (2016). 1er Estudio de Educación en Línea en México 2016. AMIPCI. https://irp-cdn. multiscreensite.com/81280eda/files/ uploaded/Estudio de Educacion en Linea 2016.pdf

Asociación de Internet.mx (2017). $13^{\circ}$ Estudio sobre los Hábitos de los Usuarios de Internet en México 2017. https://irpcdn.multiscreensite.com/81280eda/ files/uploaded/13 Estudio Habitos del Usuario 2017.pdf

Avitia, P., y Uriarte, I. (2017). Evaluación de la habilidad digital de los estudiantes universitarios: Estado de ingreso y potencialeducativo. EDUTEC: Revista Electrónica de Tecnología Educativa, (61), a366. https://doi.org/10.21556/ edutec.2017.61.861

Bautista, M. G., Martínez, A. R., e Hiracheta, R. (2014). El uso de material didáctico $\mathrm{y}$ las tecnologías de información y comunicación (TIC's) para mejorar el alcance académico. Ciencia $y$ Tecnología, 14, 183-194.

Calzada, R. D. L. C., Muñoz, R. J., Ramírez, M. T., y Álvarez, F. J. (2014). Guía metodológica de virtualización de materias para ambientes virtuales de aprendizaje del nivel medio superior y superior de la Universidad de Guanajuato. Universidad de Guanajuato, Dirección de Asuntos Académicos.

Carrera, F. X., Vaquero, E., y Balsells, M. (2011). Instrumento de Evaluación de competencias digitales para adolescentes en riesgo social. EDUTEC: Revista Electrónica de Tecnología Educativa (35), a154. https://doi.org/10.21556/ edutec.2011.35.410

Casanova, I., Canquiz, L., Paredes, Í., e Inciarte, A. (2018). Visión general del enfoque por competencias en Latinoamérica. Revista de Ciencias Sociales (Ve), XXIV(4), 114-125.

Castañeda, L. J. (2009). Las universidades 
apostando por las TIC: Modelos y paradojas de cambio institucional. EDUTEC: Revista electrónica de tecnología educativa, (28), a105. $\quad$ https://doi.org/10.21556/ edutec.2009.28.453

Cejas, M. F., Rueda, M. J., Cayo, L. E., y Villa, L. C. (2019). Formación por competencias. Reto de la Educación Superior. Revista de Ciencias Sociales (Ve), $X X V(1), 94-104$.

Centro Bachillerato Tecnológico Agropecuario 197 - CBTA 197 (2018). Plan Académico de Mejora Continua. Diagnóstico para la elaboración del programa de desarrollo institucional. Centro Bachillerato Tecnológico Agropecuario 197 (Documento interno).

Creative Research Systems (2012). The Survey System. Creative Research Systems. http://www.surveysystem. com/sscalc.htm\#one

De Ibarrola, M. (2020). Los Centros de Bachillerato Tecnológico Agropecuario y la producción agrícola escolar en la formación para el trabajo. Revista Mexicana de Investigación Educativa, 25(84), 91-119.

Fajardo, I., Villata, E., y Salmerón, L. (2016). ¿Son realmente tan buenos los nativos digitales? Relación entre las habilidades digitales y la lectura digital. Anales de Psicología, 32(1), 89-97. $\quad$ http://dx.doi.org/10.6018/ analesps.32.1.185571

Felpeto-Guerrero, A., Rey-Iglesia, R., Fernández-Vázquez, A., y GarroteYáñez, D. (2015). Uso de plataformas e-learning y alfabetización digital en formación profesional a distancia. Revista de Estudios e Investigación en Psicología y Educación, (13), 163-167. https://doi.org/10.17979/ reipe.2015.0.13.490
Fernández, J. M., y Torres, J. A. (2015). Actitudes docentes y buenas prácticas con TIC del profesorado de educación permanente de adultos en Andalucía. Revista Complutense de Educación, 26, 33-49. https://doi.org/10.5209/ rev RCED.2015.v26.43812

Fragozo, G., y Gámez, H. (2020). Percepción docente sobre las tecnologías de información y comunicación como recurso instruccional, Revista Orbis, 46(16), 31-44.

Gallardo, E. E. (2012). Hablemos de estudiantes digitales y no de nativos digitales UT. Revista de Ciències de l'Educació, (1), 7-21. https://doi. org/10.17345/ute.2012.1.595

Gisbert, M., y Esteve, F. (2011). Digital Learners: La competencia digital de los estudiantes universitarios. $L a$ Cuestión Universitaria, (7), 48-59.

Hernández, R. M. (2017). Impacto de las TIC en la educación: Retos y perspectivas. Propósitos y Representaciones, 5(1), 325-347. https://doi.org/10.20511/ pyr2017.v5n1.149

Leiva, J. J., y Priegue, D. (2012). Educación Intercultural y TIC: Claves pedagógicas de la innovación y el cambio social en el siglo XXI. @ tic. Revista D'innovació Educativa, (9), 33-43. https://doi.org/10.7203/ attic.9.1950

Llomaki, L., Paavola, S., Lakkala, M., y Kantosalo, A. (2016). Digital competence - an emergent boundary concept for policy and educational research. Education and Information Technology, 21(3), 655-679. https:// doi.org/10.1007/s10639-014-9346-4

Maderick, J. A., Zhang, S., Hartley, K., y Marchand, G. (2015). Preservice teachers and self-assessing digital competence. Journal of Educational Computing Research, 
Ramírez García, Adán Guillermo; Rodríguez Sauceda, Elvia Nereyda; Pirela Hernández, Ana Arelis y Castillo Escalante, Irma Cecilia

Habilidades digitales e interés por estudiar en la modalidad E-Learning en estudiantes de

Bachillerato

54(3), 326-351.
org/10.1177/0735633115620432

Organista, J., Sandoval, M., McAnally, L., y Lavigne, G. (2016). Estimación de las habilidades digitales con propósito educativo de estudiantes de dos universidades públicas mexicanas. Revista electrónica de tecnología educativa (57), a343. https://doi. org/10.21556/edutec.2016.57.673

Oviedo, H. C., y Campo-Arias, A. (2005). Aproximación al uso del coeficiente alfa de Cronbach. Revista Colombiana de Psiquiatría, XXXIV(4), 572-580.

Panayides, P. (2013). Coephcient Alpha. Interpret with caution. Europe's Journal of Psychology, 9(4), 687-696. https://doi.org/10.5964/ejop.v9i4.653

Prendes, M. P., Gutiérrez, I., y Martínez, F. (2018). Competencia digital: Una necesidad del profesorado universitario en el siglo XXI. Revista de Educación a Distancia, 18(56), 1-22. https://revistas.um.es/red/article/ view/321591

Ramírez, A. (2017). TIC, Extensionismo y desarrollo de capacidades. Diotima. Revista Cientifica de Estudios Transdisciplinaria, 2(5), 18-34. http:// www.revista-diotima.org/documentos/ Diotima 5.pdf
Ramírez, A. G., y Cruz, J. R. (2021). Consideraciones para implementar programas de Educación Virtual. High Rate Consulting / Universidad Autónoma Chapingo.

Ramos, E. V., Otero, C. A., Heredia, F. D., y Sotomayor, G. D. S. (2021). Formación por competencias del profesional en administración: Desde un enfoque contingencial. Revista de Ciencias Sociales (Ve), XXVII(2), 451-466. https://doi.org/10.31876/rcs. v27i2.35933

Universidad Nacional Autónoma de México - UNAM (2014). Matriz de habilidades digitales. Dirección General de Cómputo y de Tecnologías de la Información y Comunicación (DGTIC), UNAM. https://educatic. unam.mx/publicaciones/matrizhabilidades-digitales-2014.pdf

Useche, M. C., y Artigas, W. (2019). Competencias de administración. Comparación de la perspectiva estudiantil gerencial. Revista Venezolana de Gerencia, 23(E-1), 384-402.

Zubieta, J., y Rama C. (Coords.) (2015). La educación a distancia en México: Una nueva realidad universitaria. Universidad Nacional Autónoma de México. 